\title{
Relação entre sistemas de medição de desempenho e projetos Seis Sigma: estudo de caso múltiplo
}

\author{
Ricardo Coser Mergulhão UFOP
}

Roberto Antonio Martins UFScar

\section{RESUMO}

O objetivo deste artigo é investigar a relação existente entre sistemas de medição de desempenho e desenvolvimento dos projetos seis sigma por meio de estudo de caso múltiplo. O relacionamento mútuo estabelecido a partir do referencial teórico foi observado na pesquisa de campo. Além disto, observou-se que existe um caráter temporal nessa relação. 0 sistema de medição de desempenho exerce influência primeiro. 0 relacionamento se dá por meio de fatores que exercem influência positiva e negativa sobre o desenvolvimento dos projetos. Já os projetos seis sigma atuam como alavancas externas na reflexão após o uso do sistema nos projetos levando a modificações no sistema de medição de desempenho.

\section{PALAVRAS-CHAVE}

sistema de medição de desempenho, medição de desempenho, Seis Sigma, melhoria contínua, medidas de desempenho.

\section{The relationship between performance measurement systems and Six Sigma projects: multiple case study}

\begin{abstract}
This paper aims to investigate the relationship between performance measurement systems and development of six sigma projects. Five case studies were carried out in Brazilian companies. The empirical findings supported the mutual relationship established after the literature review. Furthermore, a delay was observed in the relationship. The performance measurement system influences first the development of projects. There are factors which exerts both negative and positive influences on projects. In other hand, the six sigma projects play a role of external leverage during the reflection of using performance measurement system which leads to change of such systems.
\end{abstract}

\section{KEY WORDS}

performance measurement system, performance measurement, Six Sigma, continuous improvement, performance measures. 


\section{INTRODUÇÃO}

Seis Sigma e sistemas de medição de desempenho são assuntos atuais que estão sendo discutidos e pesquisados tanto por profissionais quanto na academia. Para comprovar isto, basta notar a quantidade de seminários, artigos científicos (ou não) publicados sobre esses dois temas.

O programa Seis Sigma vem atraindo a atenção pelos ganhos financeiros obtidos por algumas empresas com o desenvolvimento de projetos Seis Sigma (SANDERS; HILD, 2000; HOERL, 2001; SCHROEDER et al., 2007). No entanto, como em outras abordagens de melhoria, alguns fatores podem influenciar a escolha e o desenvolvimento dos projetos.

Paralelamente a isso, os sistemas de medição de desempenho exercem um importante papel no suporte aos programas de melhoria, atuando como barreira ou facilitador (ATTADIA; MARTINS, 2003; MARTINS; MIRANDA, 2005). Taylor e Wright (2006) destacam o papel exercido pela medição de desempenho ao suportar as atividades de melhoria contínua. Para Martins e Miranda (2005) a medição de desempenho é um elemento essencial, mas não suficiente para desenvolvimento das atividades de melhoria contínua.

Martins et al. (2006a) alegam que a falta de um sistema de medição de desempenho apropriado pode afetar tanto a eficiência quanto a eficácia dos projetos Seis Sigma, acarretando em falhas. Hwang (2006) atribui o insucesso de alguns projetos Seis Sigma à insuficiência dos dados ou coleta incompleta. Complementando, Schroeder et al. (2005) afirmam que o uso de dashboards de processo em projetos seis sigma é uma área importante para pesquisas acadêmicas.
Todavia, a revisão bibliográfica realizada apontou que praticamente não existem trabalhos acadêmicos sobre o relacionamento entre esses dois temas. Em vista disso, o objetivo deste artigo é investigar empiricamente a relação existente entre os sistemas de medição de desempenho e os projetos seis sigma.

\section{MEDIÇÃO DE DESEMPENHO}

Apesar de ser difícil encontrar uma definição única e abrangente de sistema de medição de desempenho (SMD), como Franco-Santos et al. (2007) apontaram após uma revisão bibliográfica extensa, neste artigo será adotada a de Neely (1998, p. 5) em que “... um sistema de medição de desempenho possibilita que decisões e ações sejam tomadas com base em informações porque ele quantifica a eficiência e a eficácia de decisões passadas por meio da aquisição, compilação, arranjo, análise, interpretação e disseminação de dados adequados". Tal definição associa um SMD à tomada de decisão e estabelece as atividades necessárias essenciais (aquisição, compilação, arranjo, análise, interpretação e disseminação) para o funcionamento de tal sistema. Um SMD, segundo Neely et al. (1995), pode ser observado em três níveis diferentes, ilustrados na Figura 1.

O primeiro nível é o das medidas de desempenho geralmente associadas aos objetivos e estratégia. O segundo é o agrupamento delas num conjunto de medidas de desempenho que pode constituir um SMD desde que exista uma lógica para o agrupamento e a escolha das medidas individuais. Por fim, o último nível é a interação do sistema com

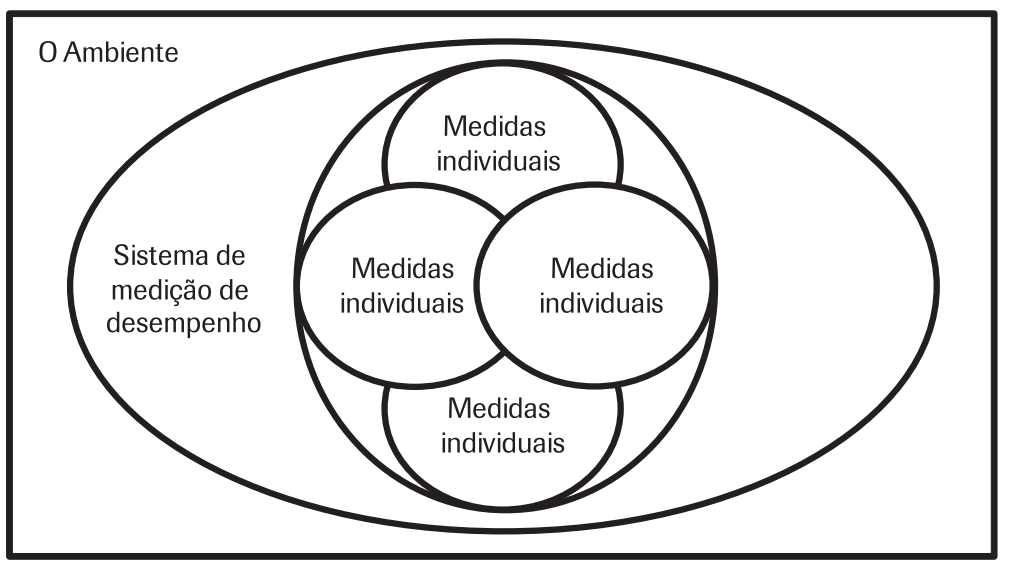

Figura 1: Elementos da medição de desempenho.

Fonte: Neely et al. (1995, p. 82) 
os ambientes interno e externo de um sistema de operações. Um aspecto importante associado à dimensão interna é o de que o SMD precisa ser consistente à cultura organizacional. Já com relação à dimensão externa, dois elementos precisam ser destacados na escolha das medidas de desempenho: os clientes e os concorrentes.

Existem dois tipos de medidas de desempenho individuais: de resultado e de processo. As primeiras retratam o resultado (objetivo) a ser atingido. Já as segundas são direcionadoras das medidas de resultado (BITITCI; NUDURUPATI, 2002).

Os SMDs tradicionais, por serem fundamentados em técnicas e métodos da Contabilidade Gerencial desenvolvida no início do século XX, falham em apoiar a consecução dos objetivos estratégicos das empresas e não promovem melhoramento contínuo sustentável (BITITCI et al., 1997). Martins (1998) e Neely (1999) destacam diversos problemas decorrentes da utilização de informações provenientes dos SMDs tradicionais.
- conferir posição pelo estabelecimento de posição no mercado, o uso do benchmarking para comparar desempenho em relação aos concorrentes e monitorar o progresso;

- comunicar posição para divulgar o desempenho para toda a organização e os reguladores (governo, acionistas e sociedade em geral);

- confirmar prioridades para a ação e verificação da alta administração, para clarificar a tomada de decisão e como significado para gestão, controle de custos e investimento; e

- compelir o progresso como significado para motivação, comunicação das prioridades da organização e como base para recompensa.

Essas quatro razões podem ser combinadas com os três papéis (atender parâmetros inegociáveis de desempenho, verificar a saúde da organização e desafiar a estratégia), provendo uma visão ampla do uso de um SMD (NEELY, 1998).

Franco-Santos et al. (2007) apresentam as condições necessárias e suficientes para um SMD, que estão ilustradas na Figura 2. Elas são categorizadas em: características do sistema, papéis do sistema e processos do sistema.

As características e os processos apresentam tanto condições necessárias quanto suficientes. Em relação aos processos, vale salientar que os de compensação comportamental ou processos explícitos de premiação podem ser ou não desenvolvidos de acordo com outros elementos presentes na organi-

Por essa razão, os SMDs precisam acompanhar as mudanças ocorridas nas configurações e estratégias da maioria das organizações (NEELY, 1998). Além disso, o desenvolvimento de novos sistemas falha porque o foco está em "o que" medir, ao invés de "como" medir (TANGEN, 2004). Nesse sentido, para que as organizações desenvolvam SMDs adequados Neely et al. (2000) argumentam que é importante considerar quatro temas associados à medição de desempenho os quais estão interligados: desenvolvimento, implementação, uso e revisão contínua.

Para Martins (2002), o desenvolvimento de um SMD pode ser baseado no uso da informação como suporte à tomada de decisão nas atividades de planejamento, controle e melhoria do desempenho organizacional. Isso pode prevenir a falta de relevância da informação gerada pelo sistema de medição de desempenho para alguns tomadores de decisão na organização. Isso é mais relevante quando se trata do uso do sistema em atividades de melhoria como, por exemplo, em projetos seis sigma.

Existem várias razões para um sistema de medição de desempenho ser utilizado, as mais comuns são os $4 \mathrm{CPs}$ (NEELY, 1998): zação, tais como cultura organizacional.

Num sentido mais abrangente, é necessário entender um SMD como um mecanismo dinâmico que precisa ser adaptado para acompanhar as condições de mudança referentes ao ambiente (interno e externo) e à estratégia organizacional (BITITCI et al., 2000). Attadia e Martins (2003) destacam que o sistema de medição de desempenho precisa evoluir harmonicamente com a melhoria contínua para que possa, em diferentes estágios da melhoria contínua, prover o suporte adequado.

Waggoner et al. (1999) deduzem, a partir da revisão de várias teorias de mudança organizacional, quatro forças que atuam na evolução e mudança dos SMDs: (i) influências internas, por exemplo, relações de poder e interesses da coalizão dominante; (ii) influências externas, por exemplo, legislação e volatilidade do mercado; (iii) assuntos relacionados aos processos, por exemplo, formas de implementação e gestão de processos; e (iv) assuntos relacionados à transformação organizacional, por exemplo, o grau de suporte da alta administração e risco de ganho ou perda da mudança.

Em vista desse dinamismo, Kennerley e Neely (2002) propõem um processo para administrar um SMD de forma que 
ele continue fornecendo dados e informações relevantes aos tomadores de decisão de uma organização, como ilustrado na Figura 3.

O processo tem início com a existência e uso do SMD. Impulsionado por alavancas externas, esse processo leva a uma reflexão acerca do sistema existente para identificar falhas e melhorias possíveis. Em seguida, é conduzida a modificação necessária para assegurar o alinhamento do sistema com as novas contingências. Por fim, é feito um desdobramento do SMD modificado de forma que ele possa ser utilizado na gestão do desempenho da organização.

Rolstadas (1995) propõe uma maneira diferente de ver tal dinâmica considerando que um SMD pode ser dividido em uma parte duradoura e outra temporária, sendo que ambas suportam as ações de controle e melhoria, respectivamente. As medidas de desempenho que compõem a parte temporária podem migrar para a parte duradoura desde que se tornem essenciais ao acompanhamento do desempenho.

A partir de um estudo de caso múltiplo, em empresas com certificado ISO 9001, Martins e Miranda (2005) concluíram que um sistema de medição de desempenho é um elemento necessário, mas não suficiente para condução das atividades de melhoria contínua. Ele é necessário porque a falta ou inadequação dele ao estágio de melhoria contínua da organização pode dificultar as atividades de melhoria contínua. Todavia também foi observado que somente um sistema adequado não é suficiente, porque é necessário competência e motivação para usá-lo.

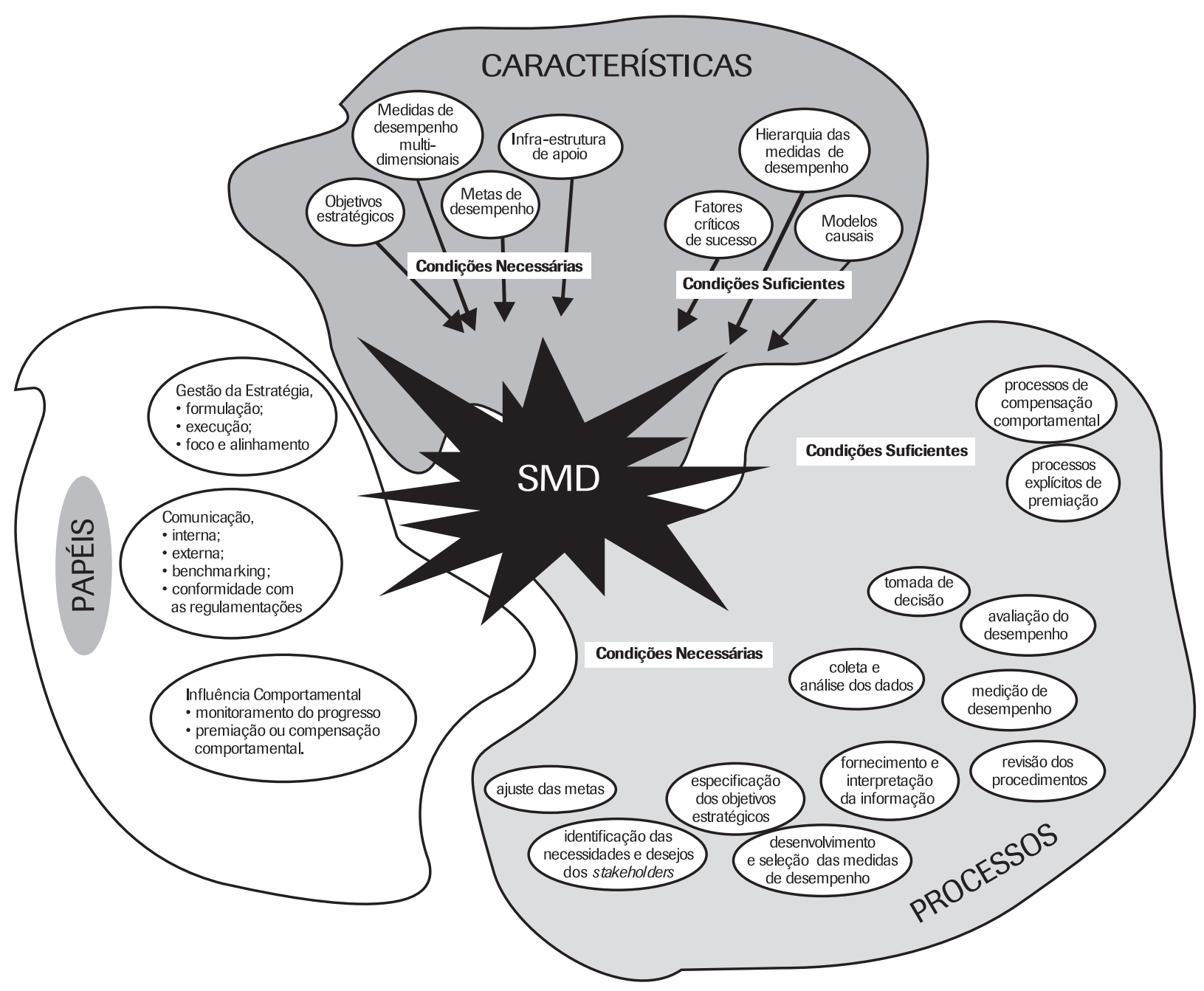

Figura 2: Grupos de características de um SMD do negócio

Fonte: Adaptado de Franco-Santos et al. (2007) 
A revisão bibliográfica efetuada sobre sistemas de medição de desempenho permitiu identificar alguns elementos que podem influenciar o desenvolvimento das atividades de melhoria. Esses elementos estão ilustrados na Figura 2 sob a forma de condições necessárias e suficientes de um SMD. Nesse sentido, o sistema de medição de desempenho pode influenciar por meio desses elementos (ou fatores) os projetos seis sigma, como está ilustrado na Figura 4.

\section{SEIS SIGMA}

Seis Sigma é um programa de melhoria da qualidade que tem por objetivo reduzir a variabilidade dos processos por meio da aplicação de métodos estatísticos e ferramentas da gestão da qualidade (GRYNA, 2001; CORONADO; ANTONY, 2002; PANDE et al., 2002). Para Schoroeder et al. (2007), os métodos e técnicas não são inéditos e já vinham sendo utilizados em outras abordagens de gestão da qualidade.

As características que distinguem Seis Sigma de outros programas de melhoria são: estrutura de treinamento; definição ampla de valor para os clientes que inclui não somente qualidade, mas também serviço e entrega; e ligação clara entre os processos a serem melhorados e os ganhos financei- ros (SCHROEDER et al., 2007; ARNHEITER e MALEYEFF, 2005; HARRY e SCHROEDER, 2000; ECKES, 2001).

As atividades de melhoria são conduzidas por projetos, denominados projetos seis sigma. Snee (2001, p. 66) define um projeto seis sigma como "um problema agendado para solução que tem um conjunto de indicadores que podem ser usados para selecionar os objetivos e metas do projeto e monitorar o progresso".

Os projetos são conduzidos por pessoas denominadas de Belts, que recebem treinamento intensivo em métodos estatísticos e ferramentas de gestão da qualidade. Dependendo do perfil, nível de treinamento e habilidades, os belts podem ser Champions, Master Black Belts, Black Belts e Green Belts. De acordo com Goldman (2005), os Belts são:

- Champion: usualmente é um dos executivos ou gerentes que apóia o programa Seis Sigma. Ele também tem a habilidade de identificar os projetos adequados, selecionar e guiar as pessoas;

- Master Black Belt: é um especialista nas técnicas e métodos estatísticos e de gestão da qualidade, e também na implementação de projetos. Ele também treina pessoas e ajuda a remover barreiras que afetam o desenvolvimento dos projetos;

- Black Belt: é um indivíduo treinado nas técnicas e métodos estatísticos e de gestão da qualidade. Ele lidera as

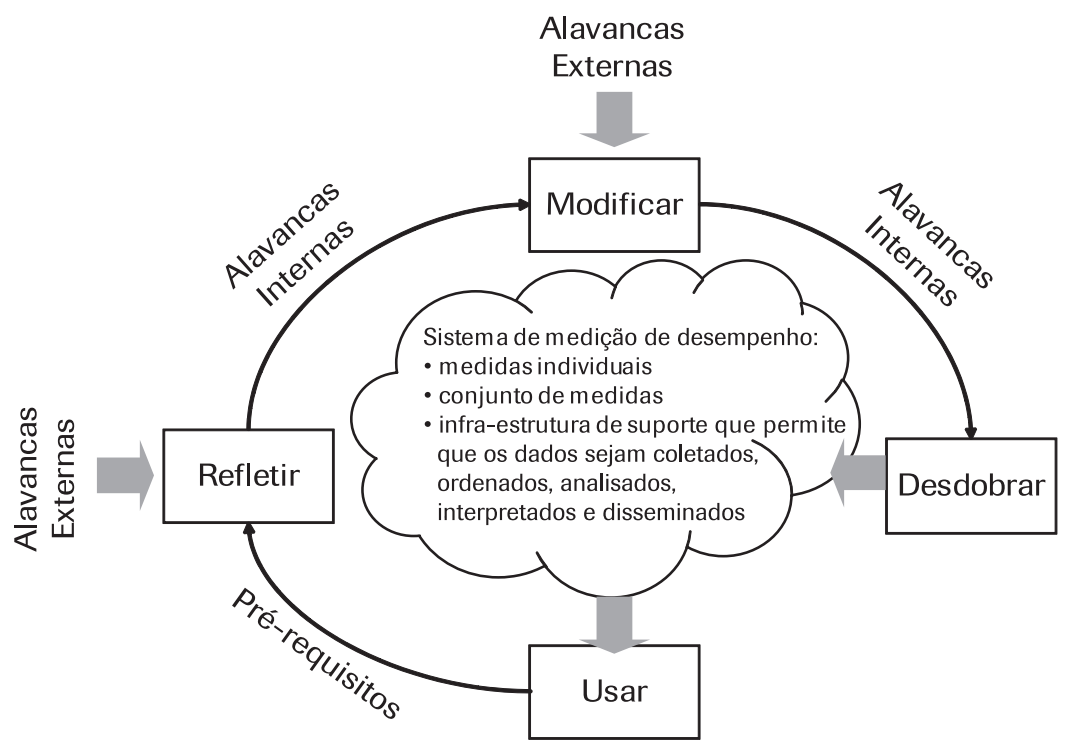

Figura 3: Fatores que afetam a evolução dos SMDs.

Fonte: Kennerley e Neely (2002, p.1241). 
equipes no desenvolvimento dos projetos e tem dedicação integral ao programa; e

- Green Belt: é um funcionário treinado em técnicas e métodos estatísticos e de gestão da qualidade com dedicação parcial que fornece suporte na implementação e aplicação dos métodos e técnicas nos projetos.

A estrutura de Belts é um diferencial do Seis Sigma em relação a outras abordagens de gestão da qualidade e permite um funcionamento mais orgânico e mecânico das atividades de melhoria (SCHROEDER et al., 2007).

Os Belts utilizam o método DMAIC (Define; Measure; Analyse; Improve; e Control) para o desenvolvimento dos projetos (CORONADO; ANTONY, 2002). Cada fase do método requer a aplicação de métodos estatísticos e ferramentas de gestão da qualidade, que não são novos, mas são revitalizados quando aplicados dentro do DMAIC (SCHROEDER et al., 2007; BREYFOGLE, 1999). No Quadro 1, o DMAIC é detalhado.

Uma atividade crítica é a seleção de projetos seis sigma. Devido à escassez de recursos financeiros e humanos, é primordial selecionar bem os projetos. Três classes de critérios podem ajudar na seleção e priorização. A primeira classe é relacionada aos resultados ou benefícios para a organização. Os critérios são impacto na satisfação dos clientes, realização da estratégia do negócio, desenvolvimento de competências fundamentais e resultados financeiros. A segunda classe é relacionada à viabilidade do projeto. Os critérios são complexidade, custo, conhecimento requerido, suporte e probabilidade de sucesso. Finalmente, a última classe relaciona-se ao impacto organizacional. Os critérios são a aprendizagem organizacional e quebra de barreiras entre os departamentos (PANDE et al., 2002).

\section{Essencialmente, o Seis Sigma é um programa de - melhoria da qualidade que tem por objetivo reduzir} a variabilidade dos processos por meio da aplicação de métodos estatísticos e ferramentas da gestão da qualidade.

Martins e Mergulhão (2006) argumentam que é importante estabelecer um relacionamento confiável entre as medidas de desempenho não-financeiras (característica crítica para qualidade do produto ou serviço) e financeiras (redução de custos e ganhos do projeto) a fim de se selecionar bem os projetos seis sigma.

Num programa Seis Sigma, são utilizadas algumas medidas de desempenho para quantificar a variabilidade dos processos e geração de defeitos ou erros (WERKEMA, 2002). A escolha da medida de desempenho está fortemente associada ao tipo de dado da característica crítica da qualidade (do inglês critical to quality - CTQ). As medidas

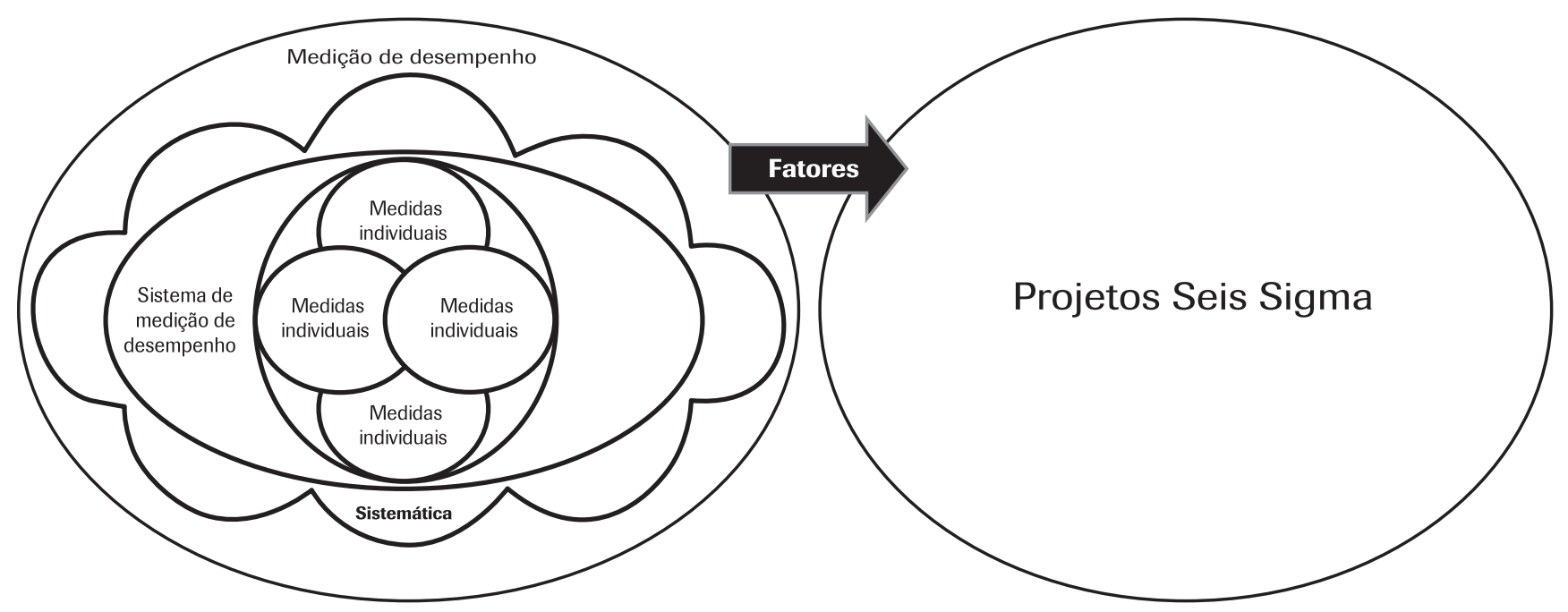

Figura 4: Visão parcial do relacionamento entre sistema de medição de desempenho e projetos seis sigma. 
de desempenho do seis sigma podem ser agrupadas em três categorias: baseadas em defeituosos, baseadas em defeitos e em indicadores de capabilidade. O Quadro 2 apresenta uma síntese de alguns das medidas de desempenho no âmbito do Seis Sigma.
A categoria dos defeituosos, de acordo com Werkema (2002), não leva em consideração o número de defeitos e é mais utilizada em situações em que qualquer defeito é sério para um dado resultado de um processo. A baseada em defeitos leva em consideração o número de defeitos. Já a dos

Quadro 1: Fases do método DMAIC.

\begin{tabular}{|c|c|}
\hline Fase & Descrição \\
\hline Define & $\begin{array}{l}\text { A equipe levantada identifica os melhores projetos seis sigma com base nos objetivos estratégicos. Após } \\
\text { isso, a equipe determina o que é crítico para qualidade (do inglês, Critical To Quality - CTQ) para os clientes. }\end{array}$ \\
\hline Measure & A equipe define os processos ligados com a CTQ, e eles medem o desempenho dos processos selecionados. \\
\hline Analyse & $\begin{array}{l}\text { Aplicando métodos estatísticos a equipe procura identificar as principais causas da variação do processo } \\
\text { que geram não-conformidades por meio de análises do desempenho do processo. A equipe confirma a } \\
\text { variável a ser melhorada. }\end{array}$ \\
\hline Improve & $\begin{array}{l}\text { A equipe conduz experimentos para estabelecer o melhor nível das variáveis identificadas na fase anterior. } \\
\text { A equipe estabelece um plano para implementar as mudanças. }\end{array}$ \\
\hline Control & $\begin{array}{l}\text { A equipe aplica técnicas e métodos estatísticos e da qualidade para garantir a estabilidade estatística do } \\
\text { processo dentro de limites aceitáveis. }\end{array}$ \\
\hline
\end{tabular}

Fonte: Adaptado de Henderson e Evans (2000).

Quadro 2: Indicadores Seis Sigma.

\begin{tabular}{|c|c|c|}
\hline Categorias & Nomenclatura & Descrição \\
\hline \multirow[t]{3}{*}{ Defeituosos } & $\begin{array}{l}\text { Proporção de } \\
\text { defeituosos (p) }\end{array}$ & $\begin{array}{l}\text { Refere-se à fração de amostras de um item que possuem um ou mais defeitos } \\
\text { (BREYFOGLE, 1999) }\end{array}$ \\
\hline & $\begin{array}{l}\text { First Throughput } \\
\text { Yield (FTY) }\end{array}$ & $\begin{array}{l}\text { Corresponde à probabilidade de se encontrar zero defeito ao se inspecionar uma } \\
\text { amostra que advém do processo (BREYFOGLE, 1999) }\end{array}$ \\
\hline & $\begin{array}{l}\text { Rolled Throughput } \\
\quad \text { Yield (RTY) }\end{array}$ & $\begin{array}{l}\text { Representa a probabilidade de um único produto passar por vários processos e sair } \\
\text { livre de defeitos (WERKEMA, 2002; PANDE et al., 2002) }\end{array}$ \\
\hline \multirow[t]{2}{*}{ Defeitos } & $\begin{array}{l}\text { Defeitos por } \\
\text { unidade (DPU) }\end{array}$ & $\begin{array}{l}\text { Reflete o número médio de defeitos, de todos os tipos, sobre o número total de } \\
\text { unidades da amostra (PANDE et al., 2002) }\end{array}$ \\
\hline & $\begin{array}{l}\text { Defeitos por milhão de } \\
\text { oportunidades (DPMO) }\end{array}$ & $\begin{array}{l}\text { Representa o número total de defeitos em um milhão de unidades produzidas } \\
\text { dividido pelo número total de oportunidades de defeito (WERKEMA, 2002) }\end{array}$ \\
\hline \multirow[t]{3}{*}{$\begin{array}{l}\text { Indicadores de } \\
\text { capabilidade }\end{array}$} & $C_{p k}$ & $\begin{array}{l}\text { Índice de capabilidade muito utilizado, pois leva em conta onde a posição da média } \\
\text { do processo está localizada em relação às especificações (MONTGOMERY, 2004). }\end{array}$ \\
\hline & $P_{p k}$ & $\begin{array}{l}\text { Sua utilização é mais adequada quando o processo não está sob controle estatístico. } \\
\text { No entanto, desde que isso seja considerado, as propriedades estatísticas dele não } \\
\text { são determináveis e conseqüentemente impossibilita que qualquer inferência válida } \\
\text { sobre seus valores populacionais seja feita (MONTGOMERY, 2004, p. 234). }\end{array}$ \\
\hline & $\begin{array}{l}\text { Níveis sigma de curto } \\
\text { e longo prazo (Zcp) e } \\
\text { (Zlp) }\end{array}$ & $\begin{array}{l}\text { Consiste em medir a distância da média à especificação mais próxima (LSE ou } \\
\text { LIE) em quantidade de desvios-padrão (sigmas), utilizando a distribuição normal } \\
\text { reduzida (z) (CARVALHO, 2002). Em função do deslocamento de } 1,5 \text { desvios- } \\
\text { padrão, considerado por algumas empresas, ele assume as formas de curto e longo } \\
\text { prazo. A última considera o deslocamento de } 1,5 \text { sigma (BREYFOGLE, 1999) }\end{array}$ \\
\hline
\end{tabular}


indicadores de capabilidade é, a princípio, aplicável apenas a variáveis contínuas, pois para as do tipo atributo (defeitos ou defeituosos) não é aplicável (CARVALHO, 2002). Porém, Pande et al. (2002) e Carvalho (2002) destacam que é possível chegar aos níveis sigma de capabilidade para variáveis do tipo atributo, pois a fração de defeituosos pode ser convertida em defeitos por milhão de oportunidades (do inglês, Defects Per Million Opportunities - DPMO) e, com isso, o caminho inverso pode ser efetuado por meio de uma tabela de conversão.

Além das medidas de desempenho do Quadro 2, como capabilidade e rolled throughput yield, Hwang (2006) também propõe tempo de ciclo e custos operacionais como medidas típicas de um programa Seis

Sigma. Esse autor ainda argumenta que elas podem ser calculadas a partir de dados disponíveis nos manufacturing execution systems. Schroeder et al. (2007) classificam de forma geral as medidas de desempenho utilizadas no Seis Sigma em medidas orientadas aos clientes e medidas financeiras em diferentes níveis hierárquicos.

A Figura 5 apresenta uma síntese da revisão bibliográfica efetuada sobre Seis Sigma. Primeiramente, tem-se o programa Seis Sigma que é composto pelos projetos, os quais são conduzidos pelos Belts aplicando o método DMAIC.

No sentido de procurar estabelecer o relacionamento entre medição de desempenho e Seis Sigma, Martins et al. (2006b) apresentam os resultados de um estudo de caso que constatou que o uso da medição de desempenho é diferente ao longo das fases do DMAIC de projetos seis sigma. Esses mesmos autores destacam que, na escolha dos projetos e na gestão da carteira de projetos, "o que medir" é uma decisão crítica porque uma medida de desempenho inapropriada ou dados não confiáveis podem conduzir erroneamente todo o projeto. No desenvolvimento dos projetos, a qualidade dos dados e "o que medir" são elementos críticos nas fases Define

\section{Em muitas situações a maturidade do sistema de medição de desempenho não é adequada ao estágio de melhoria contínua do programa Seis Sigma.}

e Measure do DMAIC. Outra observação importante é a dependência das fases Analyse e Improve das fases anteriores do DMAIC. Durante e depois da fase Control, novas medidas de desempenho podem ser adicionadas ao SMD da organização. Isto é coerente com aquilo previsto por Rolstadas (1995). Vale destacar que nada é tratado acerca da confiabilidade dos dados nos manufacturing execution systems na proposta de Hwang (2006).

A partir da revisão bibliográfica realizada pode-se deduzir a existência do relacionamento entre sistema de medição de desempenho e desenvolvimento de projetos seis sigma. $\mathrm{O}$ SMD afeta o desenvolvimento dos projetos e, por outro lado, este último também requer que o sistema atenda às necessi-

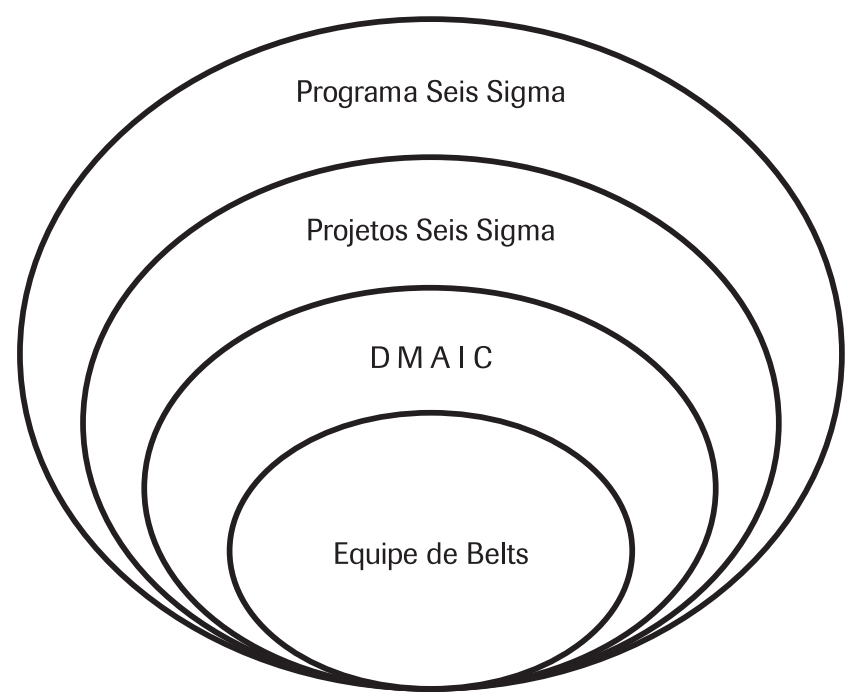

Figura 5: Visão sistêmica do Seis Sigma. 
dades de informação dos Belts da seguinte forma: existência de medidas de desempenho seis sigma; existência de um relacionamento causal detalhado, uma vez que os projetos são para melhorar os processos; informações precisas; e um relacionamento confiável entre as medidas financeiras e nãofinanceiras. Portanto, nota-se que a influência é mútua, como está representado na Figura 6.

\section{PESQUISA DE CAMPO}

A pesquisa foi conduzida em cinco organizações de indústrias diferentes que têm o programa Seis Sigma implementado. O método de pesquisa utilizado foi o do estudo de caso múltiplo do tipo explanatório, porque ele é mais adequado quando o objetivo é investigar um fenômeno contemporâneo que está sob a perspectiva dos indivíduos e o contexto das empresas. A coleta de dados aconteceu por meio de entrevistas semi-estruturadas, observações e análise de documentos, que são instrumentos de pesquisa mais adequados para a coleta de informações de caráter qualitativo (YIN, 2001). Master Black Belts, Black Belts e Green Belts das empresas pesquisadas foram entrevistados com apoio de um roteiro de entrevista que continha as principais variáveis da pesquisa.

Os critérios para a escolha do primeiro caso foram facilidade de acesso e reconhecimento formal de que a empresa tem um programa Seis Sigma implementado. No entanto, ao longo do desenvolvimento do caso, outros fatores como tipo de processo de produção (contínuo ou discreto), tempo de adoção do programa Seis Sigma e geração de bens ou serviços foram levantados pelos entrevistados como fatores importantes.

Isso conduziu a escolha dos casos subseqüentes. Segundo Yin (2001), esse processo em que um caso contribui para a escolha do próximo é conhecido como "bola de neve". Destaca-se que algumas empresas recusaram-se a participar da pesquisa por considerarem os projetos seis sigma estratégicos e sigilosos, mesmo depois de esclarecido que o foco da pesquisa não estava nas soluções técnicas desses projetos. A Tabela 1 apresenta as principais características das empresas estudadas.

\section{PRINCIPAIS EVIDÊNCIAS DA PESQUISA DE CAMPO}

Inicialmente, será apresentada a estrutura do programa Seis Sigma de cada empresa estudada. O que se pode observar, a partir dos dados da Tabela 2, é que o programa é estruturado de forma diferenciada em relação a alguns aspectos relacionados, de forma direta ou indireta, ao sistema de medição de desempenho.

As Empresas C e D implementaram o Seis Sigma por iniciativa própria. Isso, num primeiro momento, gerou um menor envolvimento da alta administração que foi aumentando à medida que os ganhos financeiros dos projetos sur-

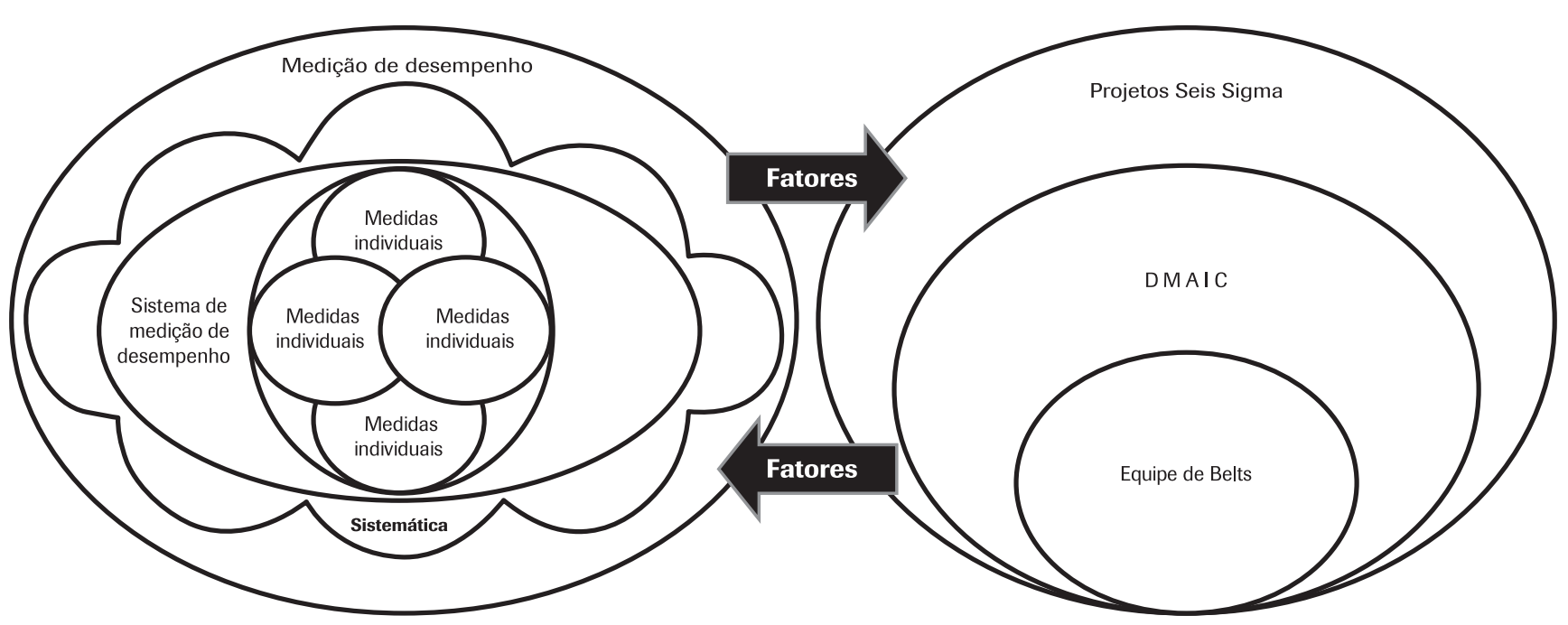

Figura 6: Relacionamento entre sistema de medição de desempenho e projetos seis sigma. 
giram. Apesar de a Empresa A ter implementado o programa de forma top-down, o envolvimento da alta administração foi menor porque se acreditava que o programa não era adequado à empresa. No entanto, após os resultados dos primeiros projetos serem divulgados, o apoio dela aumentou.
Nas Empresas B e E, o apoio da alta administração existiu desde o início.

A gestão do programa Seis Sigma parece estar relacionada com os objetivos declarados pelos entrevistados para o programa. Isto porque, naquelas em que os objetivos estavam

Tabela 1: Características gerais das empresas estudadas.

\begin{tabular}{|c|c|c|c|c|c|}
\hline & \multicolumn{5}{|c|}{ Empresa } \\
\hline & A & B & $\mathrm{C}$ & $\mathrm{D}$ & $E$ \\
\hline $\begin{array}{l}\text { Principais } \\
\text { produtos }\end{array}$ & $\begin{array}{l}\text { Produtos de } \\
\text { telecomuni- } \\
\text { cações }\end{array}$ & $\begin{array}{l}\text { Papel e } \\
\text { celulose }\end{array}$ & $\begin{array}{l}\text { Componentes para } \\
\text { linha branca }\end{array}$ & $\begin{array}{c}\text { Componentes de } \\
\text { motores para a } \\
\text { indústria automotiva }\end{array}$ & $\begin{array}{l}\text { Serviços } \\
\text { bancários }\end{array}$ \\
\hline Origem do capital & Alemã & Brasileira & Americana & Alemã & Americana \\
\hline $\begin{array}{l}\text { Tipo predominante do } \\
\text { processo de produção }\end{array}$ & $\begin{array}{l}\text { Produção } \\
\text { em massa }\end{array}$ & $\begin{array}{l}\text { Processo } \\
\text { contínuo }\end{array}$ & $\begin{array}{l}\text { Produção } \\
\text { em massa }\end{array}$ & $\begin{array}{l}\text { Produção } \\
\text { em massa }\end{array}$ & $\begin{array}{c}\text { Prestação de } \\
\text { serviço em massa }\end{array}$ \\
\hline $\begin{array}{l}\text { Certificações, } \\
\text { programas etc. }\end{array}$ & $\begin{array}{c}\text { CMMI, ISO 9001, } \\
\text { ISO 14001, TL 9000, } \\
\text { PNQ }\end{array}$ & ISO 9001 & $\begin{array}{c}\text { ISO } 9001, \text { ISO } 14001 \\
\text { OHSAS } 18001\end{array}$ & $\begin{array}{c}\text { ISO/TS 16949, ISO } \\
\text { 14001, OHSAS } \\
\text { 18001, ISO/QS } 9000\end{array}$ & ISO 9001 \\
\hline
\end{tabular}

Tabela 2: Aspectos gerais do Seis Sigma nas empresas estudadas.

\begin{tabular}{|c|c|c|c|c|c|c|}
\hline & & \multicolumn{5}{|c|}{ Empresa } \\
\hline & & A & B & $\mathrm{C}$ & $\mathrm{D}$ & $\mathrm{E}$ \\
\hline \multirow{2}{*}{$\begin{array}{c}\text { Forma inicial } \\
\text { de implementação }\end{array}$} & Top-down & $\checkmark$ & $\checkmark$ & - & - & $\checkmark$ \\
\hline & Bottom-up & - & - & $\checkmark$ & $\checkmark$ & - \\
\hline \multirow{2}{*}{$\begin{array}{c}\text { Objetivos do } \\
\text { Programa Seis Sigma }\end{array}$} & Resolução de problemas & $\checkmark$ & - & $\checkmark$ & $\checkmark$ & - \\
\hline & Execução das diretrizes estratégicas & - & $\checkmark$ & - & - & $\checkmark$ \\
\hline \multirow{2}{*}{$\begin{array}{l}\text { Gestão do programa } \\
\text { Seis Sigma }\end{array}$} & Acompanhamento de indicadores-macros & $\checkmark$ & $\checkmark$ & - & - & $\checkmark$ \\
\hline & Acompanhamento local dos projetos & $\checkmark$ & $\checkmark$ & $\checkmark$ & $\checkmark$ & $\checkmark$ \\
\hline \multirow{2}{*}{$\begin{array}{c}\text { Estrutura de } \\
\text { responsabilidades }\end{array}$} & Usual & $\checkmark$ & $\checkmark$ & $\checkmark$ & - & $\checkmark$ \\
\hline & Usual + White e Yellow Belts & - & - & - & $\checkmark$ & - \\
\hline \multirow[t]{2}{*}{ Treinamento } & Usual & - & $\checkmark$ & $\checkmark$ & $\checkmark$ & - \\
\hline & Usual + Aspectos de medição de desempenho & $\checkmark$ & - & - & - & $\checkmark$ \\
\hline \multirow{2}{*}{$\begin{array}{l}\text { Abrangência dos } \\
\text { projetos seis sigma }\end{array}$} & Parcial & $\checkmark$ & - & - & - & - \\
\hline & Toda empresa & - & $\checkmark$ & $\checkmark$ & $\checkmark$ & $\checkmark$ \\
\hline \multirow{2}{*}{$\begin{array}{l}\text { Difusão do Programa } \\
\text { Seis Sigma }\end{array}$} & Homogênea & - & $\checkmark$ & $\checkmark$ & $\checkmark$ & $\checkmark$ \\
\hline & Heterogênea & $\checkmark$ & - & - & - & - \\
\hline \multirow{2}{*}{$\begin{array}{l}\text { Estrutura de tecnologia } \\
\text { de informação }\end{array}$} & Flexível (facilidade de customização) & - & $\checkmark$ & - & - & $\checkmark$ \\
\hline & Pouco flexível & $\checkmark$ & - & $\checkmark$ & $\checkmark$ & - \\
\hline
\end{tabular}


mais relacionados com a consecução da estratégia, Emprepara o programa. Já nas Empresas C e D, cujo objetivo era resolução de problemas para redução de custos, não foram observados tais indicadores. Aparentemente a associação do programa à implementação da estratégia faz com que a alta administração se interesse mais pelo programa, utilizando-se de indicadores-macros para acompanhá-lo. Também foi constatado que, no nível de projetos seis sigma, todas as empresas apresentaram como primordiais os ganhos financeiros, enquanto que outros objetivos, como disseminação da cultura Seis Sigma, foram constatados nas Empresas A, C e D. sas B e E, foi observada a presença de indicadores-macros

A estrutura de Tecnologia de Informação (TI) apresentouse como um elemento facilitador nas Empresas B e E devido à flexibilidade dela para aprimorar o SMD e o próprio sistema de custeio à medida que projetos foram sendo desenvolvidos. Por outro lado, a falta de flexibilidade observada nas demais empresas estudadas fez com que, aparentemente, os projetos se adaptassem aos dados disponíveis, como no caso relatado por um Black Belt da Empresa A.

$\mathrm{Na}$ Tabela 3, são apresentados os relacionamentos encontrados entre o sistema de medição de desempenho e o desenvolvimento dos projetos seis sigma nas cinco empresas estudadas. A seguir são apresentados os fatores observados nos casos que expressam o relacionamento estudado.

Os sistemas de custeio parecem não s projetos Seis Sigma podem exercer o papel
de alavanca externa que leva a uma reflexão do
sistema de medição de desempenho a partir do uso
ocorrido na seleção e desenvolvimento dos projetos.

Com relação à estrutura de responsabilidades, a Empresa $\mathrm{D}$ apresentou as denominações White Belt (formado com treinamento de duração de $1,5 \mathrm{~h}$ para todos os funcionários) e Yellow Belt (formado com treinamento de duração de $12 \mathrm{~h}$ para os funcionários envolvidos mais com a coleta de dados). O objetivo foi aproximar os Belts do pessoal operacional, onde os dados para os projetos são coletados.

Quanto ao treinamento, apenas nas Empresas A e E foram incluídos tópicos relacionados à medição de desempenho no treinamento de Belts. Isso aparentemente promoveu um amadurecimento no desenvolvimento dos projetos seis sigma destas, que, apesar de apresentarem um foco estratégico para o Seis Sigma, não tiveram problemas com escopos extensos dos projetos seis sigma. $\mathrm{Na}$ Empresa $\mathrm{B}$, por exemplo, o Seis Sigma é voltado para a execução das diretrizes estratégicas, porém não apresentou treinamento com aspectos sobre a medição de desempenho em seu conteúdo.

Em quase todas as empresas pesquisadas, os projetos seis sigma abrangiam toda a organização. A exceção foi a Empresa A, cujo programa está implementado em apenas algumas áreas. A difusão dele também segue o mesmo padrão. $\mathrm{Na}$ Empresa A, existem áreas que possuem alinhamento dos projetos com a estratégia e outras que executam projetos aparentemente desvinculados dela, ou seja, não existe uma homogeneização na forma de desenvolvimento dos projetos nessa empresa, principalmente no que se refere ao processo de seleção deles. suportar as necessidades de informações exigidas pelos executores dos projetos seis sigma. Até o sistema de custeio baseado em atividades $(A B C)$, que, segundo Martins e Mergulhão (2006), parece ser adequado às necessidades dos projetos, foi apontado como falho nos casos analisados devido a direcionadores inadequados (um problema na implementação do $\mathrm{ABC}$ ) e também de apontamentos feitos incorretamente pelos usuários (um problema de confiabilidade nos dados). A Empresa A foi a única que não apresentou problemas desse tipo devido ao fato de ela possuir uma baixa quantidade de custos indiretos e, com isso, o sistema de custeio por absorção ser suficiente. Entretanto, isto merece uma atenção maior dos pesquisadores devido à ênfase em ganhos financeiros dos projetos.

A falta de medidas de desempenho cria uma dificuldade inicial logo na identificação dos projetos potenciais, na fase Define. Isso pode conduzir ao desenvolvimento de projetos voltados mais para fins de promoção pessoal, como foi observado nas Empresas A e C, do que alinhados com os objetivos da empresa. Por outro lado, a existência de medidas adequadas influencia positivamente o desenvolvimento dos projetos, pois deixa clara para belts a importância do alinhamento e da confiabilidade dos dados nos projetos.

Acerca do estabelecimento de relacionamento entre as medidas não-financeiras e as financeiras, nas Empresas A, B, $\mathrm{C}$ e E existem avalistas financeiros que se envolvem somente na validação dos ganhos no início e no término dos projetos. Na Empresa D, o avalista financeiro recebe a denominação de Money Belt - esse funcionário recebeu treinamento sobre Seis Sigma. Isso, diferentemente dos outros casos, pode proporcionar uma visão mais apurada sobre as medidas não-financeiras. A validação dessas estimativas por avalistas financeiros está em acordo com a proposta de Michalski 
(2003). No entanto, Rudisill e Clary (2004) afirmam que esses avalistas deveriam ser integrados em todas as etapas do DMAIC para que possam procurar por mais oportunidades de melhoria, o que não foi constatado nos casos estudados.

O sistema de medição de desempenho também exerce influência sobre outros fatores que afetam o desenvolvimento dos projetos seis sigma. A associação dos ganhos financeiros dos projetos às promoções dos executores fez com que existisse um maior envolvimento dos Belts nas empresas estudadas. Além disso, o apoio da alta administração ao programa Seis Sigma aumentou à medida que os ganhos financeiros alcançados pelos projetos foram divulgados. Nesta situação, o SMD passa a ser um elemento importante porque é nele que se baseiam as informações de ganho. Todavia, essa influência pode vir a ser até negativa devido ao fato de os sistemas de custeio não fornecerem informações precisas.

A busca por uma estrutura de TI adequada, em termos de flexibilidade (facilidade de customização), é importante para o estabelecimento de uma infra-estrutura para facilitar o desenvolvimento dos projetos seis sigma. Caso isso não ocorra, o desenvolvimento dos projetos pode ser prejudicado e, conforme destacado anteriormente na Empresa A, o próprio direcionamento dos projetos pode ser afetado.

Tabela 3: Fatores do relacionamento entre sistemas de medição de desempenho e projetos Seis Sigma

\begin{tabular}{|c|c|c|c|c|c|c|}
\hline & \multirow[b]{2}{*}{ Relacionamentos } & \multicolumn{5}{|c|}{ Empresa } \\
\hline & & A & B & C & $\mathrm{D}$ & $\mathrm{E}$ \\
\hline \multirow{11}{*}{$\begin{array}{c}\text { Sistema de } \\
\text { medição de } \\
\text { desempenho } \\
\text { influenciando } \\
\text { os projetos } \\
\text { Seis Sigma }\end{array}$} & $\begin{array}{l}\text { Os sistemas de custeio não atendem às necessidades de informação } \\
\text { financeira }\end{array}$ & $\checkmark$ & - & $\checkmark$ & & $\checkmark$ \\
\hline & $\begin{array}{l}\text { Faltam medidas de desempenho para a identificação dos projetos } \\
\text { Seis Sigma }\end{array}$ & & $\checkmark$ & $\checkmark$ & - & - \\
\hline & $\begin{array}{l}\text { Incluir aspectos sobre SMD nos treinamentos facilita o desenvolvimento } \\
\text { dos projetos }\end{array}$ & & - & - & - & $\checkmark$ \\
\hline & $\begin{array}{l}\text { O estabelecimento do relacionamento entre as medidas não-financeiras } \\
\text { e financeiras influencia o desenvolvimento dos projetos }\end{array}$ & - & $\checkmark$ & $\checkmark$ & & $\checkmark$ \\
\hline & A falta de confiabilidade dos dados influencia a estimativa financeira & $\sqrt{ }$ & $\sqrt{ }$ & $\checkmark$ & - & - \\
\hline & O sistema de medição de desempenho influencia outros fatores & $\sqrt{ }$ & $\checkmark$ & - & - & - \\
\hline & A estrutura de $\mathrm{TI}$ influencia o desenvolvimento dos projetos seis sigma & $\sqrt{ }$ & $\checkmark$ & - & - & - \\
\hline & $\begin{array}{l}\text { O acesso às informações não-financeiras e financeiras influencia o } \\
\text { desenvolvimento dos projetos Seis Sigma }\end{array}$ & & & - & & $\checkmark$ \\
\hline & $\begin{array}{l}\text { O hábito de se medir influencia a confiabilidade dos dados usados nos } \\
\text { projetos Seis Sigma }\end{array}$ & & - & & & \\
\hline & $\begin{array}{l}\text { O aumento do conhecimento das relações causais influencia o } \\
\text { desenvolvimento dos projetos Seis Sigma }\end{array}$ & $\checkmark$ & $\checkmark$ & $\checkmark$ & $\checkmark$ & - \\
\hline & $\begin{array}{l}\text { O detalhamento do desdobramento das diretrizes influencia o } \\
\text { desenvolvimento dos projetos Seis Sigma }\end{array}$ & - & $\checkmark$ & - & - & $\checkmark$ \\
\hline \multirow{3}{*}{$\begin{array}{c}\text { Projetos } \\
\text { Seis Sigma } \\
\text { influenciando } \\
\text { o sistema de } \\
\text { medição de } \\
\text { desempenho }\end{array}$} & $\begin{array}{l}\text { O desenvolvimento dos projetos seis sigma promove um uso mais } \\
\text { analítico do sistema de medição de desempenho }\end{array}$ & - & & & & \\
\hline & $\begin{array}{l}\text { A autonomia dos Belts promove uma estruturação do sistema de } \\
\text { medição de desempenho }\end{array}$ & - & - & - & $\checkmark$ & $\checkmark$ \\
\hline & $\begin{array}{l}\text { A disseminação dos conceitos do Seis Sigma ajuda a promover um hábito } \\
\text { de se medir }\end{array}$ & - & - & - & & - \\
\hline
\end{tabular}


Nas Empresas A, B e D, o acesso às informações atuou como um fator facilitador. Na Empresa E, ocorreram restrições na obtenção de informações sobre custos por serem considerados sigilosos, ou seja, o acesso às informações funcionou como um fator inibidor. Já nas Empresas D e E, o apoio do Champion foi considerado fundamental para que o acesso a esse tipo de informações fosse garantido. $\mathrm{O}$ acesso às informações, quando atuando como inibidor, pode prejudicar tanto a eficiência (aumentando o tempo de desenvolvimento dos projetos) quanto a eficácia dos projetos (atingir os ganhos estabelecidos). ders e Hild (2001) alertam que os responsáveis pela coleta dos dados passam a distorcê-los quando existe um clima de medo e punição.

Observou-se nas empresas estudadas, com exceção da Empresa E, que o aumento do conhecimento das relações causais entre as medidas resultado e processo promove mudanças no sistema de medição de desempenho. Isso pode conduzir à formação de séries históricas que podem ajudar no desenvolvimento dos projetos seis sigma. No entanto, vale destacar que, com exceção da Empresa B, em alguns casos, esse aumento de entendimento causal ficou restrito aos controles locais sem ser repassado para o SMD, limitando o enriquecimento da estrutura do sistema.

Na Empresa B, foi verificado que o desdobramento das diretrizes, que resultam em projetos seis sigma, parece estar relacionado aos escopos extensos dos projetos. Por outro lado, a Empresa E, que também desdobra projetos a partir das diretrizes, tal problema não foi observado. A implementação mais es-

Nas empresas estudadas, com exceção da Empresa B, foi observado que a falta do hábito de se medir prejudica a confiabilidade dos dados usados nos projetos. O que parece ter sido favorável na Empresa B foi o tipo de processo de produção que, por ser contínuo com monitoramento intenso e, com isso, o hábito de se medir já era difundido. Nas demais empresas, os esforços de coleta de dados ad-hocs foram freqüentemente citados pelos entrevistados, sendo que os motivos para sua realização eram a inexistência de dados ou a falta de confiabilidade dos existentes. A falta de acesso aos dados ou de confiabilidade deles não foi considerada por Hwang (2006) em sua proposta de utilização dos dados dos manufacturing execution sytems. Todavia os casos estudados mostraram que esses fatores são importantes. Na Empresa D, a disseminação dos conceitos Seis Sigma para todos na empresa, conforme citado pelos entrevistados, ajudou no comprometimento das pessoas, garantindo uma maior confiabilidade dos dados devido aos cuidados na coleta.

Na Empresa D, um projeto foi desenvolvido e uma medida de desempenho, associada à CTQ melhorada, foi acompanhada durante 12 meses e, após isso, foi descartada. Logo depois, essa medida voltou a ser acompanhada e uma nova coleta ad-hoc precisou ser realizada. Isto pode ser explicado porque não foi considerada a possibilidade que Rolstadas (1995) aventa de tornar as medidas temporárias do projeto medidas de rotina após a conclusão do projeto. $\mathrm{Na}$ Empresa $\mathrm{C}$, devido à pressão por resultados e um sentimento de punição caso eles não fossem alcançados, existia uma confiabilidade muito baixa nos dados do SMD. San- truturada do Seis Sigma na Empresa B começou a ocorrer em 2005, enquanto que na Empresa E se iniciou em 2001. Além disso, na Empresa E existe um Black Belt especialista em Gestão pelas Diretrizes para acompanhar o desdobramento em projetos. Esses dois fatores podem explicar as diferenças encontradas. Vale ressaltar que para Linderman et al. (2003) as metas, além de aspectos técnicos, envolvem aspectos comportamentais e quando elas são percebidas como muito difíceis pelas pessoas pode ocorrer um baixo compromisso que pode conduzir a um baixo desempenho. Por outro lado, Gryna (2001) afirma que a fase Measure serve para ajustar o projeto à medida que os resultados dessa fase ajudam a constatar que ele é muito ambicioso e necessita ser dividido em outros projetos. Essa seria uma solução para os projetos com escopos muito amplos.

Além dos elementos relacionados ao SMD que influenciam os projetos seis sigma, foi constatado que os últimos também influenciam os primeiros. Nas empresas estudadas, com exceção da Empresa A, os projetos seis sigma influenciam a análise crítica das medidas de desempenho uma vez um Green Belt afirmou que passou a considerar a variabilidade das medidas em suas análises. Nesse sentido, de acordo com o observado na Empresa B, o DMAIC reforça o uso do SMD para melhoria e controle.

No sentido de se alcançar essa maturidade do uso do SMD, foi observado, nas Empresas D e E, que a autonomia dos Belts é um fator fundamental para que isto ocorra uma vez que eles influenciam no desenvolvimento do sistema. Vale salientar que na Empresa A, devido à baixa confiabili- 
dade nos dados, alguns projetos foram abortados e uma ação de reestruturação no SMD foi tomada. Isto demonstra que a influência é mútua.

Finalmente, a partir dos dados da pesquisa de campo foi elaborada a Tabela 4, que estabelece algumas necessidades e usos do SMD nos projetos seis sigma. A partir dos dados da Tabela 4, pode-se observar que cada fase do DMAIC requer características específicas do SMD. Além disso, constatou-se que o uso do sistema, segundo os 4 CPs de Neely (1998), é diferente em cada fase do DMAIC.

\section{CONCLUSÕES}

O presente artigo teve como objetivo investigar a relação existente entre sistemas de medição de desempenho e desenvolvimento dos projetos seis sigma por meio de estudo de caso múltiplo. O relacionamento mútuo estabelecido a partir do referencial teórico foi confirmado na pesquisa de campo. A seguir são levantados três pontos que permitem entender melhor como ocorre tal relacionamento.

O primeiro ponto é a característica temporal do relacionamento. Foi observado que o SMD influencia primeiro o desenvolvimento dos projetos, pois existem fatores que influenciam a seleção e as primeiras fases do DMAIC. Com o projeto em desenvolvimento ou com alguns encerrados, então, a influência em sentido oposto começa a ocorrer. Os dados da Tabela 4 reforçam isto.

O relacionamento acontece por meio de fatores. A Tabela 3 contém aqueles que foram observados na pesquisa de campo. Eles reforçam o relacionamento mútuo estabelecido na revisão teórica. Além disso, pode-se observar que existem mais fatores relacionados ao SMD que aos projetos seis sigma.

Os fatores não atuam todos da mesma forma como foi observado nos casos estudados. A pesquisa permitiu também caminhar no sentido de entender a qualificação do relacionamento, isto é, como os fatores atuam. Foi observado que existem fatores que atuam de forma negativa e outros de forma positiva. Alguns deles acabam dificultando a seleção e o desenvolvimento dos projetos. Vale salientar que a influência é menor nas fases Improve e Control. Já outros fatores atuam como alavancas, principalmente no desenvolvimento. Os fatores negativos podem ser transformados em fatores positivos desde que exista uma adequação do SMD. Isto ocorre por influência dos projetos seis sigma.

Inclusive vale destacar que o sistema de custeio por atividades que era esperado exercer uma influência positiva acabou por não exercer tal papel nas Empresas B, C, D e E. De maneira diferente da esperada, o sistema de custeio por absorção acabou exercendo influência positiva na Empresa A. Isto merece mais investigações para elucidar o tipo de influência que o sistema de custeio pode exercer. Isto é tão importante que foi observado o papel de avaliadores para os projetos e do Money Belt, especificamente na Empresa D.

Vários fatores relativos aos sistemas de medição de desempenho foram observados na pesquisa de campo exercendo influência negativa quando, na verdade, era esperado o contrário. Um exemplo disto é a confiabilidade dos dados. A falta dela atua negativamente porque é preciso fazer coleta de dados ad-hoc. Isto acarreta, no mínimo, um aumento do tempo de desenvolvimento dos projetos. Por outro lado, a existência dela pode aumentar o uso do sistema. Aliás, as co-

Tabela 4: Necessidades e uso da medição de desempenho nos projetos Seis Sigma.

\begin{tabular}{|c|c|c|}
\hline Fase & Necessidades em relação à medição de desempenho & Uso \\
\hline Define & $\begin{array}{l}\text { Medidas de desempenho estratégicas } \\
\text { Relacionamento causal entre as medidas de desempenho } \\
\text { Medidas de desempenho confiáveis } \\
\text { Sistema de custeio adequado } \\
\text { Facilidade de acesso às informações não-financeiras e financeiras }\end{array}$ & Confirmar prioridades \\
\hline Measure & $\begin{array}{l}\text { Medidas de desempenho confiáveis } \\
\text { Facilidade de acesso às informações não-financeiras e financeiras }\end{array}$ & Conferir a posição \\
\hline $\begin{array}{l}\text { Analyse e } \\
\text { Improve }\end{array}$ & $\begin{array}{l}\text { Medidas de desempenho confiáveis } \\
\text { Relacionamento causal entre as medidas de desempenho } \\
\text { Facilidade de acesso às informações não-financeiras e financeiras }\end{array}$ & Compelir o progresso \\
\hline Control & $\begin{array}{l}\text { Medidas de desempenho confiáveis } \\
\text { Sistema de custeio adequado } \\
\text { Estrutura de TI flexível (fácil customização) }\end{array}$ & Comunicar posição \\
\hline
\end{tabular}


letas de dados ad-hoc não são necessariamente uma influência negativa do SMD no desenvolvimento dos projetos. Elas podem acontecer por dois motivos inerentes aos projetos: investigação de variáveis novas que não são acompanhadas pelo sistema atual da organização e estudo de novas relações causais entre variáveis de processo.

Todavia, isto não é necessariamente um indicativo de um desvio da teoria. O que aconteceu é que em muitas situações a maturidade do sistema de medição de desempenho não é adequada ao estágio de melhoria contínua do programa Seis Sigma. Portanto, a falta de harmonia entre o SMD e os projetos seis sigma fez com que os fatores atuem de forma negativa. Isto explica em parte como o desenvolvimento dos projetos exerce influência sobre os SMDs.

Aqui surge o terceiro ponto a ser destacado. Os projetos seis sigma podem exercer o papel de alavanca externa que leva a uma reflexão do sistema de medição de desempenho a partir do uso ocorrido na seleção e desenvolvimento dos projetos. Isto pode requerer modificação no SMD, como é previsto por Kennerly e Neely (2002). Isto ocorre quando a maturidade do sistema não é adequada à necessidade de uso requerida pelos belts, como é previsto por Attadia e Martins (2003) ao defenderem que o SMD, para suportar a atividade de melhoria contínua, precisa estar adequado ao nível de maturidade desta última.

Isto ocorre na forma de incorporação de novas medidas de desempenho ao SMD da organização porque o acompanhamento feito na fase Control precisa continuar mesmo após o término do projeto seis sigma, como foi observado na Empresa D. Contudo, pode ser necessário mudar o sistema para adequá-lo ao estágio de melhoria do Seis Sigma, o que seria importante na Empresa B, por exemplo. Outra forma de influência observada foi a melhoria do uso dos dados em que a formação de belt ajudou a elaborar análises mais sofisticadas sobre o desempenho. Na revisão bibliográfica realizada, nada foi encontrado acerca da análise de adequação do sistema de medição de desempenho ao programa Seis Sigma. Isto merece mais atenção dos pesquisadores para evitar que um SMD influencie negativamente o desenvolvimento dos projetos.

Portanto, o relacionamento entre sistemas de medição de desempenho e projetos seis sigma tem um caráter dinâmico que é exercido por fatores que atuam negativa e positivamente. A atuação negativa de alguns fatores deve-se à falta de harmonia entre o sistema de medição de desempenho e o estágio de melhoria contínua dos projetos seis sigma.

\section{Artigo recebido em 27/06/2007 Aprovado para publicação em 26/03/2008}

\section{REFERÊNCIAS}

ARNHEITER, E. D.; MALEYEFF, J. The integration of lean management and six sigma. The TQM Magazine, v. 17, n. 1, p. 5-18, 2005.

ATTADIA, L. C. L.; MARTINS, R. A. Medição de desempenho como base para evolução da melhoria contínua. Revista Produção, v. 13, n. 2, p. 33-41, 2003.

BITITCI, U. S. Dynamics of performance measurement systems. International Journal of Operations \& Production Management, v. 20, n. 6, p. 692-704, 2000.
BITITCI, U. S.; NUDURUPATI, S. S. Driving continuous improvement. Manufacturing Engineer, v. 81, n. 5, p. 230-235, 2002.

BITITICI, U. S.; CARRIE, A. S.; McDEVITT, L. Integrated performance measurement systems: a development guide. International Journal of Operations \& Production Management, v. 17, n. 17, p. 522-534, 1997.

BREYFOGLE, F. W. Implementing Six Sigma: Smarter Solutions Using Statistical
Methods. New York: John Wiley and Sons, 1999.

CARVALHO, M. M. Selecionando projetos Seis Sigma. In: ROTONDARO, R. G. (Coord.). Seis Sigma: estratégia gerencial para a melhoria de processos, produtos e serviços. São Paulo: Atlas, p. 49-79, 2002.

CORONADO, R. B.; ANTONY, J. Critical success factors for the successful implementation of Six Sigma projects in organisations. The TQM Magazine, v. 14, n. 2, p. 92-99, 2002. 
ECKES, G. A revolução do Seis Sigma: o método que levou a GE e outras empresas a transformar processos em lucros. 3. ed., Rio de Janeiro: Campus, 2001.

FRANCO-SANTOS, M.; KENNERLEY, M.; MICHELI, P.; MARTINEZ, V.; MASON, S.; MARR, B.; GRAY, D.; NEELY, A. Towards a definition of a business performance measurement system. International Journal of Operations \& Production Management, v. 27, n. 8, p. 784-801, 2007.

GOLDMAN, H. H. The origins and development of quality initiatives in American business. The TQM Magazine, v. 17, n. 3, p. 217-225, 2005.

GRYNA, F. M. Quality Planning \& Analysis. New York: McGraw-Hill, 2001.

HARRY, M.; SCHROEDER, R. Six sigma: the breakthrough management strategy revolutionising the world's top corporations. New York: Currency Publishers, 2000.

HENDERSON, K.; EVANS, J. Successful implementation of six sigma: benchmarking General Electric Company. Benchmarking, v. 7, n. 4, p. 260-281, 2000.

HOERL, R. W. Six sigma black belts: what do they need to know? Journal of Quality Technology, v. 33, n. 4, p. 391-406, 2001.

HWANG, Y-D. The practices of integrating manufacturing execution systems and six sigma methodology. International Journal of Advanced Manufacturing Technology, v. 31, p. 145-154, 2006.

KENNERLEY, M.; NEELY, A. A framework of the factors affecting the evolution of performance measurement systems. International Journal of Operations \& Production Management, v. 22, n. 11, p. 1222-1245, 2002.

LINDERMAN, K.; SCROEDER, R. G.; SRILATA, Z.; CHOO, A. S. Six sigma: a goal-theoretic perspective. Journal of Operations Management, v. 1, n. 11, p. 330-341, 2003.
MARTINS, R. A. Sistemas de medição de desempenho: um modelo para estruturação do uso, Tese (Doutorado em Engenharia de Produção), Escola Politécnica. São Paulo: Universidade de São Paulo, 1998.

Uso da informação sobre desempenho como direcionador de projeto de sistemas de medição de desempenho. Anais. XXII Encontro Nacional de Engenharia de Produção - ENEGEP. Curitiba, PR, 2002.

MARTINS, R. A.; MERGULHÃO, R. C. Financial and non-financial performance measures in Six Sigma initiatives. Proceedings. Third International Conference on Production Research - Americas' Region, Curitiba, PR, Brazil, PUC, 2006.

MARTINS, R. A.; MERGULHÃO, R. C.; LEAL, J. B. S. O papel dinâmico da medição de desempenho nos projetos Seis Sigma: Um estudo de caso. Anais. XXVI Encontro Nacional de Engenharia de Produção - ENEGEP. Fortaleza, CE, 2006a.

MARTINS, R. A.; MIRANDA, R. A. M. Factors Affecting the Support of Performance Measurement to Continuous Improvement Activities, Proceedings. 6th CINet Conference, Brighton, CENTRIM/CINet, 2005.

MARTINS, R. A; MERGULHÃO, R. C.; LEAL, J. B. S. The use of performance measurement in the Six Sigma projects: a case study. Proceedings. The Fifth International Conference on Performance Measurement, University of Cambridge, UK, p. 489-496, 2006b.

MICHALSKI, W. J. Six Sigma Tool Navigator: The Master Guide for Teams. New York: Productivity Press, 2003.

MONTGOMERY, D. C. Introdução ao controle estatístico da qualidade. 4. ed. Rio de Janeiro: LTC, 2004.

NEELY, A. Measuring business performance. London: The Economist Books, 1998.

The performance measurement revolution: why now and what next? International Journal of Operations \& Production Management, v. 19, n. 2, p. 205-228, 1999.

NEELY, A.; GREGORY, M.; PLATTS, K. Performance measurement system design a literature review and research agenda. International Journal of Operations \& Production Management, v. 15, n. 4, p. 80-116, 1995.

NEELY, A.; MILLS, J.; PLATTS, K.; RICHARDS, H.; GREGORY, M.; BOURNE, M.; KENNERLEY, $M$. Performance measurement system design: developing and testing a process-based approach. International Journal of Operations \& Production Management, v. 20, n. 10, p. 1119-1145, 2000.

PANDE, P. S.; NEUMAN, R. P.; CAVANAGH, R. R. Estratégia Seis Sigma: como a GE, a Motorola e outras grandes empresas estão aguçando seu desempenho. Rio de Janeiro: Qualitymark, 2002.

ROLSTADAS, A. Performance management: $A$ business process benchmarking approach. New York: Chapman \& Hall, 1995.

RUDISILL, F.; CLARY, D. The management accountant's role in Six Sigma. Strategic Finance, v. 86, n. 5, p. 34-39, 2004.

SANDERS, D.; HILD, C. R. Common myths about Six Sigma. Quality Engineering, v. 13, n. 2, p. 269-276, 2001.

Six sigma on business processes: common organizational issues. Quality Engineering, v. 12, n. 4, p. 603-610, 2000.

SCHROEDER, R. G.; LINDERMAN, K.; LIEDTKE, C.; CHOO, A. S. Six sigma: definition and underlying theory. Journal of Operations Management, 2007. doi:10.1016/j. jom.2007.06.007.

SCHROEDER, R. G.; LINDERMAN, K.; ZHANG, D. Evolution of quality: first fifty issues of Production and Operations Management. Production and Operations Management, v. 14, n. 4, p. 468-481, 2005. 
SNEE, R. D. Dealing with the Achilles' hell of six sigma initiatives - Project selection is key to success. Quality Progress, v. 34, n. 3, p. 66-72, 2001.

TANGEN, S. Performance measurement: from philosophy to practice. International Journal of Productivity and Performance Management, v. 53, n. 8, p. 726-737, 2004.
TAYLOR, W. A.; WRIGHT, G. H. The contribution of measurement and information infrastructure to TQM success. Omega, v. 34, n. 4 , p. 372-384, 2006.

WAGGONER, D.B.; NEELY, A.D. \& KENNERLEY, M.P. The forces that shape organisational performance measurement systems: an interdisciplinary review. International
Journal of Production Economics, v. 60-61, p. 53-60, 1999

WERKEMA, M. C. C. Criando a cultura Seis Sigma. Rio de Janeiro: Qualitymark, 2002.

YIN, R. K. Estudo de caso - planejamento e métodos. 2. ed. Porto Alegre: Bookman, 2001.

\section{AGRADECIMENTOS}

Agradecemos ao Conselho Nacional de Desenvolvimento Científico e Tecnológico (CNPq) pelo apoio financeiro para desenvolvimento da pesquisa e à Coordenação de Aperfeiçoamento de Pessoal de Nível Superior (Capes) pela bolsa de estudos concedida.

\section{SOBRE OS AUTORES}

\section{Ricardo Coser Mergulhão}

Departamento de Ciências Exatas e Aplicadas

Universidade Federal de Ouro Preto

Rua 37, n.115 - Loanda - 35930-970 - João Monlevade - MG - Brasil - Caixa-Postal 24

Tel.: (31) 3852-8709 Fax: (31) 3852-8709

E-mail: mergulhao@decea.ufop.br

\section{Roberto Antonio Martins}

Departamento de Engenharia de Produção

Universidade Federal de São Carlos

End.: Rod. Washington Luís, Km 235 - Jardim Guanabara - 13565-905 - São Carlos - SP - Caixa Postal 676

Telefone: (16) 3351-8237 Fax: (16) 3351-8240

E-mail: ram@dep.ufscar.br 